\title{
ANALYTICAL CALCULATION OF THE COUPLING COEFFICIENT OF A WAVEGUIDE-CAVITY COUPLING SYSTEM
}

\section{J. Gao, LAL, B.P. 34, 91898 Orsay cedex, France}

\section{Abstract}

By applying the perturbation method, we show in a general way how to determine the coupling coefficient of a waveguide cavity coupling system and as a special example we derive the analytical expressions of the coupling coefficient for a waveguide cavity coupling system as shown in Fig. 1. The term "cavity" in this paper stands for the cavity in both standing wave and travelling wave structures.

\section{INTRODUCTION}

In the design and construction of klystrons and linear accelerating structures the coupling systems shown in Fig. 1 [1][2] and Fig. 2 are the most frequently used ones. The cavities can be standing wave or travelling wave ones. To determine the coupling coefficient between the waveguide and the cavity one can use different methods such as experiments, numerical simulations, and analytical calculations. In this paper we will show in a general way how to determine the coupling coefficient of a waveguide cavity coupling system by using a perturbation method. As an example a coupling system shown in Fig. 1 will be investigated in detail. The analytical treatment for the coupling system shown in Fig. 2 has been given in refs. [3] [4] and checked experimentally [5].

\section{GENERAL THEORY}

In a rectangular waveguide of width $a$ and height $b$ the normalised $H_{n m}$ modes can be derived from the scalar function [6]:

$$
\begin{gathered}
\psi_{n m}(x, y)=\left(\frac{\epsilon_{0 n} \epsilon_{0 m}}{a b j k_{0} Z_{0} \Gamma_{n m} k_{c, n m}^{2}}\right)^{1 / 2} \times \\
\cos \left(\frac{n \pi}{a} x\right) \cos \left(m \pi \frac{2 y-b}{b}\right)
\end{gathered}
$$

according to the following relations:

$$
\begin{gathered}
\mathbf{h}_{z n m}=\mathbf{a}_{z} k_{c, n m}^{2} \psi_{n m} \\
\mathbf{h}_{n m}=-\Gamma_{n m} \nabla_{t} \psi_{n m} \\
\mathbf{e}_{n m}=\frac{j k_{0} Z_{0}}{\Gamma_{n m}}\left(\mathbf{a}_{x} \mathbf{a}_{y}-\mathbf{a}_{y} \mathbf{a}_{x}\right) \cdot \mathbf{h}_{n m} \\
\mathbf{E}_{n m}^{ \pm}=\mathbf{e}_{n m} e^{\mp \Gamma_{n m} z} \cdot \mathbf{h}_{n m} \\
\mathbf{H}_{n m}^{ \pm}=\left( \pm \mathbf{h}_{n m}+\mathbf{h}_{z n m}\right) e^{\mp \Gamma_{n m} z}
\end{gathered}
$$

where $\epsilon_{0 n}=1(n=0)$ and $\epsilon_{0 n}=2(n>0), Z_{0}=$ $120 \pi(\Omega), k_{c, n m}^{2}=(m \pi / b)^{2}+(n \pi / a)^{2}-k_{0}^{2}, \mathbf{h}_{n m}$ and $\mathbf{e}_{n m}$ are the transverse magnetic and electric normal mode fields, $\Gamma_{n m}^{2}=k_{c, n m}^{2}-k_{0}^{2}, k_{0}=2 \pi / \lambda$, and the $\lambda$ is the wavelength in free space. The normalisation in eq. 1 has been chosen so that

$$
\int_{0}^{a} \int_{0}^{b} \mathbf{e}_{n m} \times \mathbf{h}_{n m} \cdot \mathbf{a}_{z} d x d y=1
$$

Now we consider the case of a waveguide (see Fig. 3) excited by an aperture (see Fig. 4) which can be equivalent to an electric dipole $\mathbf{P}$ and a magnetic dipole $\mathbf{M}$. The scattered field can be expanded in terms of the normal waveguide modes as follows [6]:

$$
\begin{aligned}
\mathbf{E}_{s} & =\sum a_{n} \mathbf{E}_{n}^{+} & & z>0 \\
\mathbf{H}_{s} & =\sum a_{n} \mathbf{H}_{n}^{+} & & z>0 \\
\mathbf{E}_{s} & =\sum b_{n} \mathbf{E}_{n}^{-} & & z<0 \\
\mathbf{H}_{s} & =\sum b_{n} \mathbf{H}_{n}^{-} & & z<0
\end{aligned}
$$

where the expansion coefficients $a_{n}$ and $b_{n}$ can be obtained by the following relations according to Lorentz reciprocity principle [6]:

$$
\begin{aligned}
& 2 \boldsymbol{a}_{n}=j \omega\left(\mu_{0} \mathbf{H}_{n}^{-} \cdot \mathbf{M}-\mathbf{E}_{n}^{-} \cdot \mathbf{P}\right) \\
& 2 b_{n}=j \omega\left(\mu_{0} \mathbf{H}_{n}^{+} \cdot \mathbf{M}-\mathbf{E}_{n}^{+} \cdot \mathbf{P}\right)
\end{aligned}
$$

where $\omega=\frac{k_{0}}{c}$, the electric and magnetic dipoles can be estimated as follows [6]:

$$
\begin{gathered}
\mathbf{P}=-\frac{\pi l_{1}^{3}\left(1-e_{0}^{2}\right)}{3 E_{0}\left(e_{0}\right)} \epsilon_{0} \mathbf{E}_{0} \\
\mathbf{M}_{1}=\frac{\pi l_{1}^{3} e_{0}^{2}}{3\left(K\left(e_{0}\right)-E_{0}\left(e_{0}\right)\right)} \mu_{0} \mathbf{H}_{1} \\
\mathbf{M}_{2}=-\frac{\pi l_{1}^{3} e_{0}^{2}\left(1-e_{0}^{2}\right)}{3\left(E_{0}\left(e_{0}\right)-\left(1-e_{0}^{2}\right) K\left(e_{0}\right)\right)} \mu_{0} \mathbf{H}_{2} \\
K\left(e_{0}\right)=\frac{\pi}{2}\left(1+\left(\frac{1}{2}\right)^{2} e_{0}^{2}+\left(\frac{1 \cdot 3}{2 \cdot 4}\right)^{2} e_{0}^{4}+\cdots\right) \\
E\left(e_{0}\right)=\frac{\pi}{2}\left(1-\left(\frac{1}{2}\right)^{2} e_{0}^{2}-\left(\frac{1 \cdot 3}{2 \cdot 4}\right)^{2} \frac{e_{0}^{4}}{3}-\cdots\right) \\
\epsilon_{0}=\left(1-\frac{l_{2}^{2}}{l_{1}^{2}}\right)^{1 / 2}
\end{gathered}
$$

where $\epsilon_{0}$ is the permittivity of the vacuum, and $\mu_{0}$ is the permeability of the vacuum. When the aperture is circular of radius $r$, eqs. 14 and 15 can be simplified as follows:

$$
\mathbf{P}=-\frac{2}{3} r^{3} \epsilon_{0} \mathbf{E}_{0}
$$




$$
\mathbf{M}_{1,2}=\frac{4}{3} r^{3} \mu_{0} \mathbf{H}_{1,2}
$$

As a special example we consider the $H_{10}$ mode in the waveguide. The normalised mode functions for the $H_{10}$ mode are:

$$
\begin{gathered}
\mathbf{E}_{10}^{+}=\mathbf{e}_{10} e^{-\Gamma_{10} z} \\
\mathbf{E}_{10}^{-}=\mathbf{e}_{10} e^{\Gamma_{10} z} \\
\mathbf{H}_{10}^{+}=\left(\mathbf{h}_{10}+\mathbf{h}_{z 10}\right) e^{-\Gamma_{10} z} \\
\mathbf{H}_{10}^{-}=\left(-\mathbf{h}_{10}+\mathbf{h}_{z 10}\right) e^{\Gamma_{10} z} \\
\mathbf{e}_{10}=-j k_{0} Z_{0}\left(\frac{2}{j a b k_{0} Z_{0} \Gamma_{10}}\right)^{1 / 2} \sin \left(\frac{\pi x}{a}\right) \mathbf{a}_{y} \\
\mathbf{h}_{10}=\Gamma_{10}\left(\frac{2}{j a b k_{0} Z_{0} \Gamma_{10}}\right)^{1 / 2} \sin \left(\frac{\pi x}{a}\right) \mathbf{a}_{x} \\
\mathbf{h}_{z 10}=\left(\frac{2}{j a b k_{0} Z_{0} \Gamma_{10}}\right)^{1 / 2} \frac{\pi}{a} \cos \left(\frac{\pi x}{a}\right) \mathbf{a}_{z} \\
\Gamma_{10}=k_{0}\left(1-\left(\frac{\lambda}{2 a}\right)^{2}\right)^{1 / 2}
\end{gathered}
$$

The peak power of the $H_{10}$ mode inside the waveguide can be expressed as:

$$
\begin{gathered}
P_{\text {max }}=\frac{a b Z_{0} k_{0}}{4 \Gamma_{10}} H_{x, 10, \text { max }}^{2} \\
H_{x, 10, \text { max }}=\frac{a \Gamma_{10}}{\pi} H_{z, 10, \text { max }}
\end{gathered}
$$

where $H_{x, 10, \text { max }}$ and $H_{z, 10, \text { max }}$ are the peak magnetic fields of the $H_{10}$ mode in the $x$ and $z$ directions, respectively. If the waveguide is excited by an magnetic dipole of an elliptical aperture as shown in Fig. 4 one can find from eqs. 12 and 13 the expansion coefficients $a_{1}$ and $b_{1}$ corresponding to $H_{10}$ mode as follows:

$$
\begin{gathered}
2 a_{1}=j \omega \mu_{0} \mathbf{H}_{10}^{-} \cdot \mathbf{M}=j \omega \mu_{0} \mathbf{h}_{z 10} \cdot \mathbf{M} \\
2 b_{1}=j \omega \mu_{0} \mathbf{H}_{10}^{+} \cdot \mathbf{M}=j \omega \mu_{0} \mathbf{h}_{z 10} \cdot \mathbf{M}=2 \boldsymbol{a}_{1}
\end{gathered}
$$

If one side of the waveguide is terminated with a metallic wall located at a distance of $\mathrm{L}$ from the center of the coupling aperture the forward and the backward waves will be added up travelling towards the other side of the waveguide. Due to the boundary condition set by the above mentioned metallic wall one knows that the $H_{z, 10}$ on the surface of the metallic wall is zero.

With the above preparation one can start to establish the analytical formulae of the coupling coefficient of the waveguide cavity coupling system shown in Fig. 1. According to the definition one has

$$
\beta=\frac{P}{P_{0}^{*}}
$$

where $P$ is the power radiated into the waveguide from the cavity through the coupling aperture, $P_{0}^{*}=P_{0}+U v_{g} / h$, $P_{0}$ is the power dissipated on the coupler cavity wall, $U$ is the energy stored inside the coupler cavity, and $v_{g}$ is the group velocity of the structure (the case of $v_{g}=0$ corresponds to standing wave cavity). In reality, if the power taken by the accelerated beam, $P_{b}$, is comparable to $P_{0}+U v_{g} / h$, one has to add $P_{b}$ to $P^{*}$. By using eqs. 30 and 34 one finds:

$$
\beta=\frac{\pi^{2} N Z_{0} k_{0} \Gamma_{10} e_{0}^{4} l_{1}^{6} e^{-2 \alpha d} \sin ^{2}\left(\frac{2 \pi L}{\lambda_{g, 10}}\right)}{9 a b\left(K\left(e_{0}\right)-E\left(e_{0}\right)\right)^{2}}\left(\frac{\pi}{a \Gamma_{10}}\right)^{2} \frac{H_{1}^{2}}{P_{0}^{*}}
$$

where $N$ is the number of the waveguides connected to the coupler cavity (assuming that the magnetic field on each coupling aperture is the same), $H_{1}$ is magnetic field at the location of the coupling aperture before the aperture is opened as shown in Fig. 4 (the formula corresponding to the case of $\mathrm{H}_{2}$ is omitted), $\lambda_{g, 10}$ is the waveguide wavelength of the $H_{10}$ mode, $d$ is the average wall thickness between the inner surface of the waveguide and the inner surface of the coupler cavity, and $\alpha$ is the attenuation coefficient which can be expressed as $\alpha=\frac{2 \pi}{\lambda}\left(\left(\frac{\lambda}{4 l_{1,2}}\right)^{2}-1\right)^{1 / 2}$ depending on the wave inside the aperture is $H_{10}$ or $H_{01}$ like. If we use the analytical expressions for $P_{0}$ and $U_{0}$ of $\mathrm{TM}_{010}$ mode in a pill-box cavity as what we have done in ref. [4], eq. 35 can be further simplified:

$\beta=\frac{N \pi Z_{0} k_{0} \Gamma_{10} l_{1}^{6} e_{0}^{4} e^{-2 \alpha d} \sin ^{2}\left(\frac{2 \pi L}{\lambda_{g}, 10}\right)\left(\frac{\pi}{a \Gamma_{10}}\right)^{2}}{9 a b R R_{s}(R+h)\left(K\left(e_{0}\right)-E\left(e_{0}\right)\right)^{2}\left(1+\frac{Z_{0} R v_{g}}{2 R_{s} c(R+h)}\right)}$

where $R_{s}$ is the metal surface resistance. If the aperture is circular with radius $r$ the attenuation coefficient should be expressed as $\alpha=\frac{2 \pi}{\lambda}\left(\left(\frac{\lambda}{3.41 r}\right)^{2}-1\right)^{1 / 2}$.

\section{EXAMPLE}

In this section we consider a coupling system of type I shown in Fig. 1 with two waveguides $(\mathrm{N}=2)$ to avoid the unsymmetrical fields in the cavity. The advantages of this coupling system over that of type II ( $\mathrm{N}=2$ also) are that the two waveguides can be combined more compactly into one input waveguide on the one hand and the coupling strength can be adjusted by changing the short circuit distance on the other hand. As an example, considering an S-band travelling wave structure with $h=0.02, R=0.04$, $v_{g} / c=0.03, a=0.072, b=0.02, \lambda=0.1, N=2$, $d=0.0022, L=\lambda_{g, 10} / 4$, and $2 l_{2}=h$, one gets from eq. 36 that for $\beta=1$ the coupling aperture's length $2 l_{1}$ should be 0.035 which is very close to the HFSS simulation result [7].

\section{CONCLUSIONS}

In this paper we have shown in a general way how to establish the coupling coefficient between a waveguide and a cavity. This method can be used either for the fundamental mode or higher order modes [8], and the waveguide can be either a rectangular or a circular one. As a special 
example to demonstrate the procedure we have established the analytical expression of the coupling coefficient for a waveguide cavity coupling system shown in Fig. 1.

\section{ACKNOWLEDGEMENT}

I thank J. Le Duff for his constant encouragement and showing interest to this work.

\section{REFERENCES}

[1] A.N. Parvenov, N.P. Sobenin, and B.V. Zverev, "Analytical calculation of a coupler for the linear collider accelerating section”, EPAC94, p. 2022.

[2] S.V. Ivanov, et al., "DEAY linear collider accelerating section coupler”, EPAC94, p. 2025.

[3] J. Gao, "Analytical formula for the coupling coefficient $\beta$ of a cavity-waveguide coupling system", Nucl. Instr. and Methods, A309 (1991), p. 5.

[4] J. Gao, "Analytical Approach and scaling laws in the design of disk-loaded travelling wave accelerating structures", Particle Accelerators, Vol. 43(4) (1994), p. 235.

[5] H. Bluem, et. al., "Measurements on the frequency effects and coupling of slots and irises for the APT CCDTL", PAC99, New York (1999), p. 3570.

[6] R.E. Colling, "Field theory of guided waves", McGraw-Hill (1960).

[7] P. Avrakhov, "Simulation of TW buncher for CTF3", LAL/SERA internal report, Setp. 2000.

[8] J. Gao, "On the higher order mode coupler design for damped accelerating structures”, PAC95, May 1-5, Dallas, USA (1995), p. 1717.

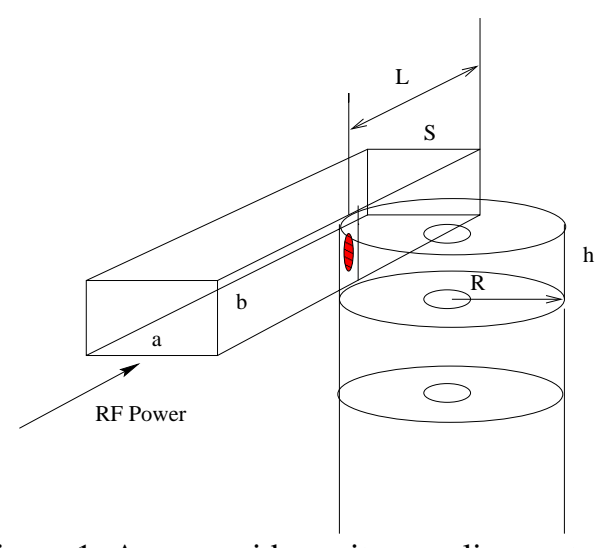

Figure 1: A waveguide cavity coupling system type I.

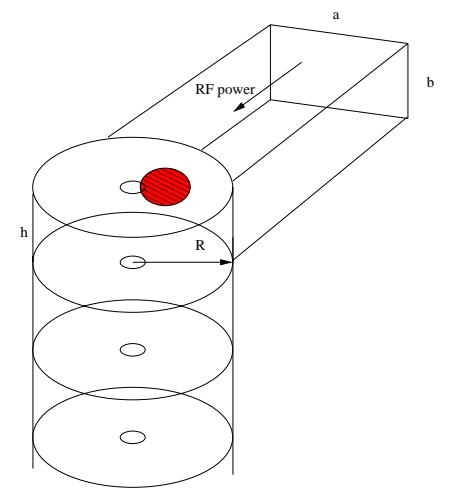

Figure 2: A waveguide cavity coupling system type II.

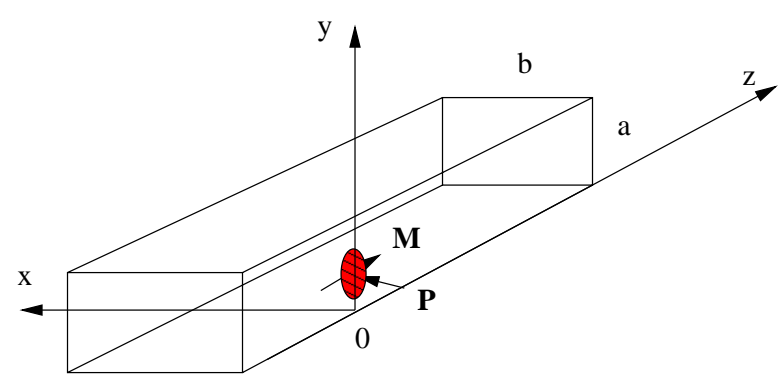

Figure 3: Waveguide excited by an electric dipole and a magnetic dipole.

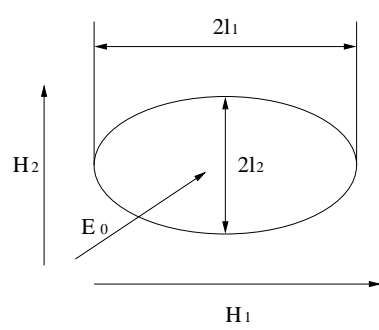

Figure 4: The elliptical coupling aperture with magnetic fields parallel to the surface and the electric field perpendicular to the surface. 